\title{
Levé bathymétrique de la retenue de Pareloup
}

\section{Bathymetric survey of Pareloup reservoir}

\section{Hervé Pujo *}

- Électricité de France, Direction des Études et Recherches. Département LNH. 6 Quai Watier, F-78401 Chatou Cedex, France.

Résumé. - Afin de mettre à jour les courbes de capacité de Pareloup, et de disposer d'une cartographie des isobathes, un relevé bathymétrique a été réalisé à Pareloup en octobre 1988. Ce levé bathymétrique a été effectué à partir d'un bateau pneumatique (zodiac MARK III) sur lequel était embarqué un sondeur à ultra-son ATLAS KRUPP DESO 20, bifréquence (33 $\mathrm{kHz}$ et $210 \mathrm{kHz}$ ) et un calculateur HP 9807. La position du bateau était repérée avec un théodolithe télémétrique AGA 140.

Mots-clés. - bathymétrie, courbe de capacité, courbes isobathes, échosondeur, Pareloup, théodolithe.

Abstract. - A bathymetric survey was carried out at Pareloup in October 1988 with a view to updating Pareloup capacity curves and to mapping the isobaths. The survey was conducted from a rubber boat (Zodiac MARK III), equipped with an ATLAS KRUPP DESO 20 ultra-sound depth finder, set for two frequencies ( $33 \mathrm{kHz}$ and $210 \mathrm{kHz}$ ), and an HP 9807 computer. Bearings of the boat were taken with an AGA 140 telemetric theodolite.

Key words. - bathymetry, capacity curve, isobath, echo sounder, Pareloup, theodolite.

\section{INTRODUCTION}

La retenue de Pareloup a été mise en eau en 1950 et la connaissance que nous avons des fonds immergés date de cette époque.

Dans le cadre général de l'étude de l'écosystème de la retenue de Pare- loup, nous avons procédé à une bathymétrie, qui est l'étude des reliefs immergés, avec un double objectif :

- mettre à jour les courbes de capacités, exprimant le volume et la surface à une profondeur donnée, afin de bien dimensionner le maillage des modèles mathématiques; 
- disposer d'une cartographie des isobathes, indispensable à la compréhension des circulations internes de masses d'eau étudiées au cours de plusieurs campagnes de traçages.

\section{PRINCIPE DE LA BATHYMÉTRIE}

La bathymétrie, qui étudie les reliefs immergés, s'apparente à la topographie terrestre. Cependant le levé bathymétrique a lieu "en aveugle", ce qui implique une méthodologie particulière.

L'acquisition des profondeurs se fait à partir d'une embarcation qui navigue en suivant une série de routes parallèles entre elles et perpendiculaires aux lignes de niveaux, appelées les profils. La finesse de représentation des fonds dépendra de l'espacement entre les profils, et sera déterminée par l'objectif de l'étude: sûreté de la navigation, envasement d'une retenue, connaissance du profil moyen d'une rivière etc...

La mesure de la profondeur d'eau est basée sur celle de la propagation dans l'eau d'une impulsion ultra-sonore. Cette impulsion, émise en surface par un sondeur, est réfléchie par le fond. Connaissant le temps de parcours (aller et retour) de limpulsion et la vitesse de propagation du son dans l'eau, il est possible d'en déduire la profondeur d'eau, appelée sonde.

Les sondeurs utilisés peuvent émettre une ou plusieurs fréquences. Chaque fréquence renvoie un écho différent selon l'obstacle rencontré; ainsi, un appareil multifréquence permet de mieux repérer le relief immergé. D'autre part, plusieurs fréquences garantissent une sécurité dans l'acquisition des mesures et apportent plus de précision.

Pour nos besoins en matière de bathymétrie, nous avons choisi un sondeur hydrographique bifréquence (basse fréquence et haute fréquence). La basse fréquence (environ $30 \mathrm{kHz}$ ) traverse les fonds très mous et se réfléchit sur les fonds durs, alors que la haute fréquence (environ $200 \mathrm{kHz}$ ) se réfléchit sur la vase. La différence de pénétration entre les deux faisceaux permet ainsi de connaître la nature des fonds et éventuellement l'épaisseur de la vase molle (fig. 1).

Les faisceaux émis se propagent dans l'eau selon un cône d'émission dont l'angle au sommet est de $10^{\circ}$ environ pour la haute fréquence et $20^{\circ}$ environ pour la basse fréquence. Si l'instant de départ de l'impulsion est parfaitement connu, l'instant de son retour est mal défini, en raison de la dispersion du faisceau et des différents chemins parcourus. L'appareil prend en compte l'instant de retour correspondant à l'arrivée du premier signal réfléchi par le fond, qui mesure la distance la plus courte entre le fond et le transducteur. Cette disposition permet, sur fond plat, de fournir une mesure correcte même si le faisceau n'est pas vertical (à cause du roulis du bateau par exemple). Par contre, sur les fortes pentes, la profondeur mesurée est minorée, car le signal le plus rapide provient du bord du cône qui rencontre le fond le premier, c'est- 


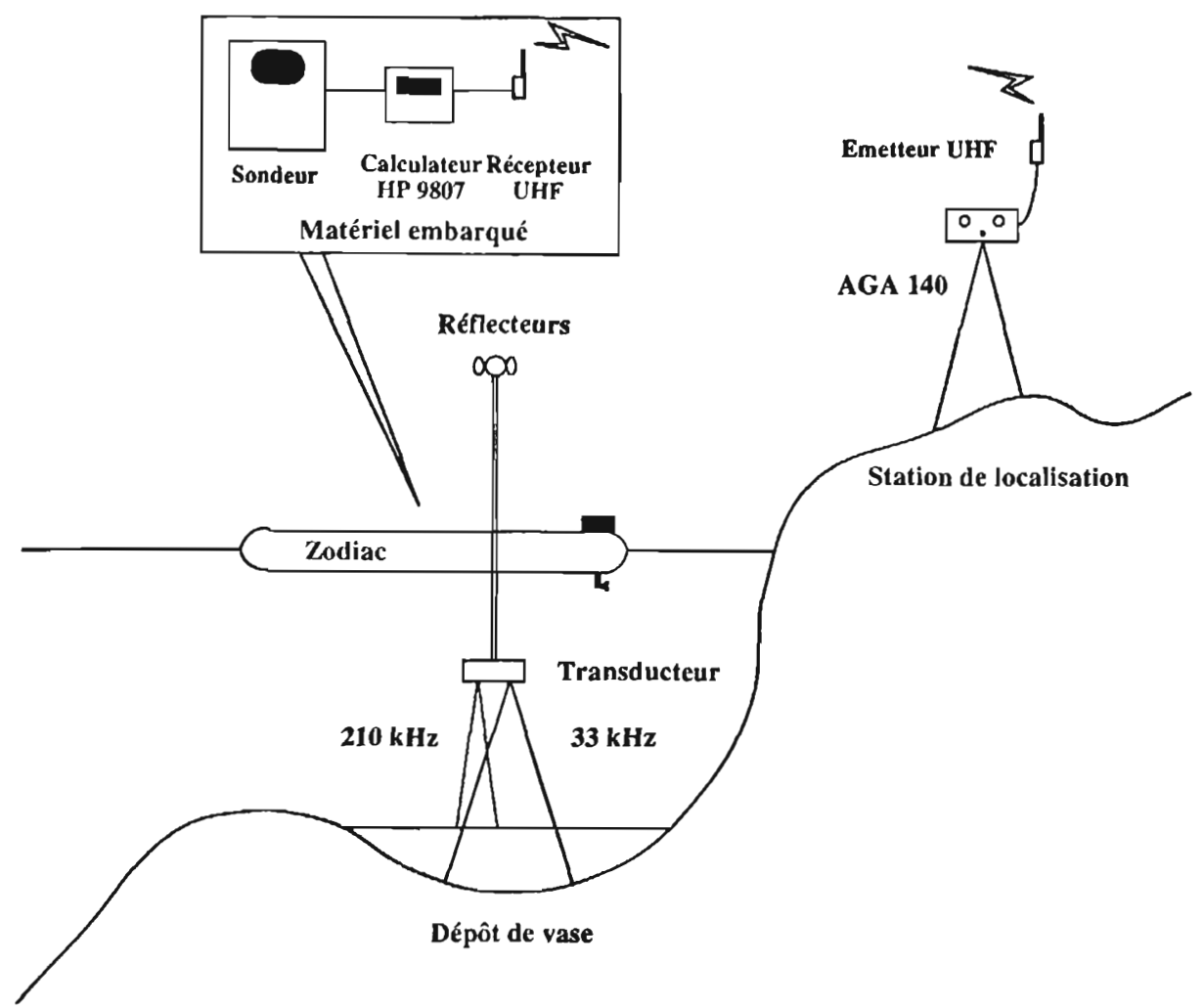

Fig. 1. - Représentation schématique du matériel utilisé pour une bathymétrie.

Fig. 1. - Materials used for bathymetrics surveys.

à-dire, d'un point situé légèrement audessus du point visé.

Afin de minimiser l'erreur de mesure, on doit, dans ce cas, effectuer les relevés en suivant des routes perpendiculaires aux isobathes. Ainsi le bateau se déplaçant dans le sens de la plus forte pente, la distance parcourue horizontalement entre l'émission et la réception du signal permet une localisation de la sonde plus représentative de la profondeur d'eau. Actuellement, un traitement du signal est intégré aux sondeurs modernes pour corriger les erreurs systématiques.

La vitesse du son dans l'eau, dépendant de la température, est mesurée in situ au début du sondage à l'aide d'un transducteur d'étalonnage et vérifiée en fin de sondage. II est préférable, pour la précision de la mesure, que la masse d'eau sondée soit isotherme et que sa température varie peu au cours du sondage. Toutefois, lorsque la zone à sonder est profonde ou que les caractéristiques de la masse d'eau sont susceptibles de va- 
rier verticalement, il faudra déterminer la valeur moyenne verticale de la vitesse du son. On peut alors procéder en intégrant verticalement l'écho renvoyé tous les mètres par une barre métallique que l'on immerge de la surface jusqu'au fond (avec un maximun de $25 \mathrm{~m}$ ).

Pendant l'opération de sondage, l'embarcation est localisée en plan par différents moyens que l'on adapte aux conditions de travail : poursuite optique, radiolocalisation ou moyens satellitaires (GPS).

Les résultats sont en permanence enregistrés par un calculateur embarqué. Les mesures de profondeur sont acquises environ quatre fois par seconde pour chaque fréquence, alors que la position du bateau est connue toutes les 1,5 seconde pour une localisation avec un théodolithe AGA. Compte tenu d'une vitesse de progression du bateau de l'ordre de $1 \mathrm{~m} \cdot \mathrm{s}^{-1}$, l'acquisition de la mesure de profondeur a lieu tous les $0,25 \mathrm{~m}$ parcourus horizontalement et celle de la position tous les $1,5 \mathrm{~m}$. Au dépouillement, la localisation de chaque sonde est calculée par interpolation linéaire entre deux positions mesurées de l'embarcation.

\section{LA BATHYMÉTRIE DU LAC DE PARELOUP}

Étant donné la forme complexe du lac de Pareloup, et la précision que I'on recherchait, cette bathymétrie a demandé un important travail préparatoire, en particulier pour la localisation.
Au préalable, une cartographie de "profils théoriques" a été établie. Ces "profils théoriques" sont destinés à servir de guide à la navigation. Ils ont été établis à partir d'un agrandissement à l'échelle 1/10000 de la carte IGN (1/25000) puis digitalisés. Afin de couvrir toute la superficie de la retenue, 180 profils, espacés de $100 \mathrm{~m}$, ont été nécessaires.

\section{III.1 Le matériel utilisé}

Pour cette bathymétrie, nous avons utilisé

- un bateau pneumatique (zodiac MARK III) ;

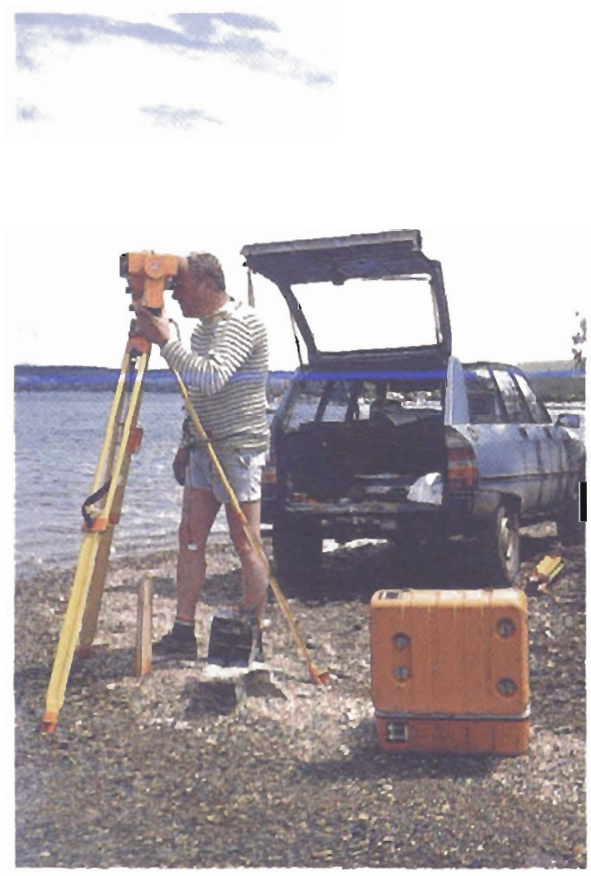

Photo 1. - Localisation avec le théodolithe télémétrique AGA 140.

Photo 1. - Localization using the AGA 140 telemetric theodolite. 

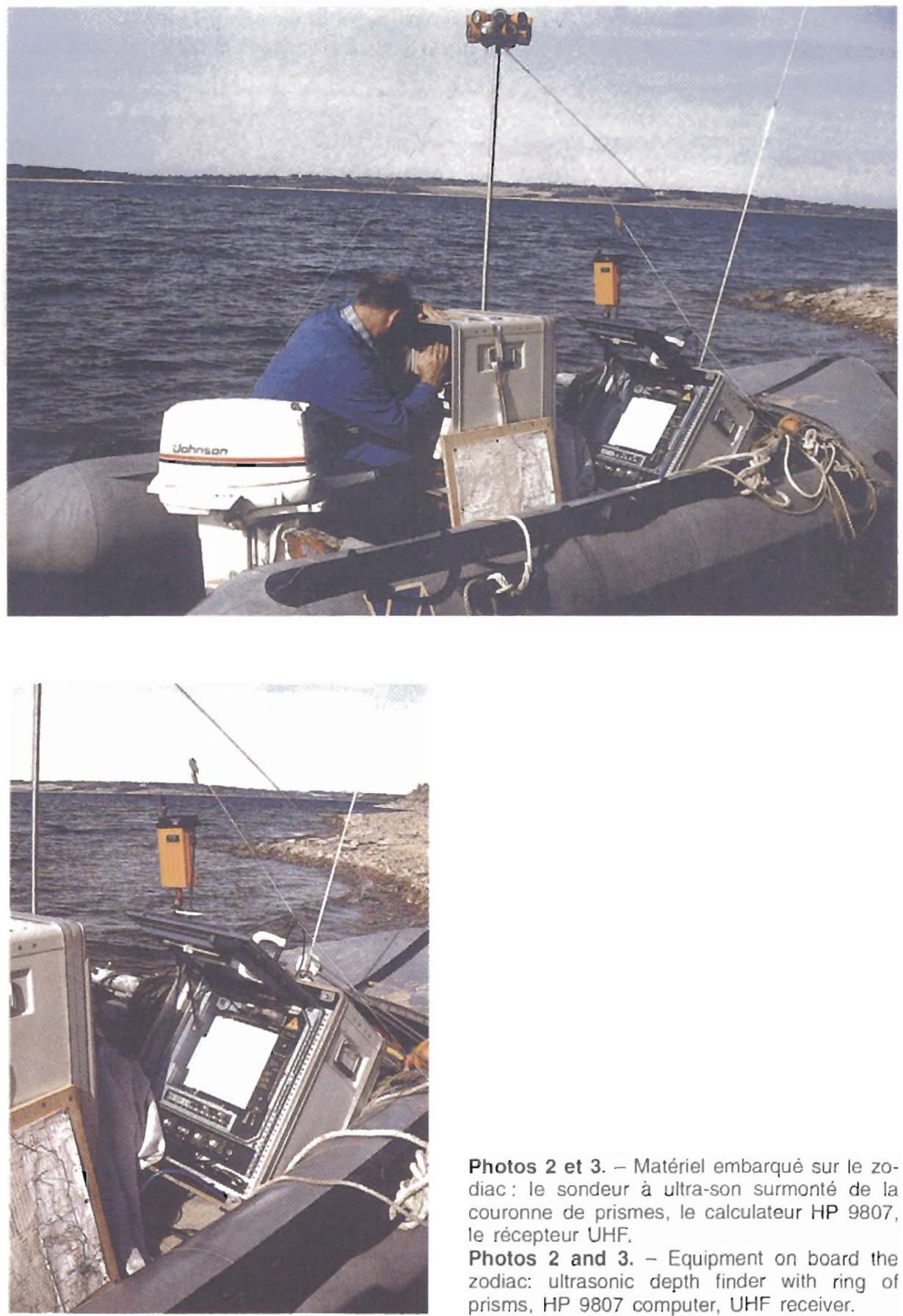

Photos 2 et 3. - Matériel embarqué sur le zodiac: le sondeur à ultra-son surmonté de la couronne de prismes, le calculateur HP 9807 , le récepteur UHF:

Photos 2 and 3. - Equipment on board the zodiac: ultrasonic depth finder with ring of prisms, HP 9807 computer, UHF receiver. 
- un sondeur à ultra-son ATLAS KRUPP DESO 20, bifréquence (33 $\mathrm{kHz}$ et $210 \mathrm{kHz}$ ) ;

- un calculateur HP 9807 ;

- un théodolithe télémétrique AGA 140.

\section{III.2 La localisation}

Pour le positionnement, nous avons opté pour la poursuite optique de l'embarcation de sondage, à partir d'une station à terre.

L'appareil utilisé est un théodolithe télémétrique $A G A 140$, qui émet un rayon infra-rouge. L'opérateur vise une couronne de prismes fixée sur la base de transducteurs du sondeur. La visée fournit mécaniquement les an- gles horizontaux et verticaux, tandis que la distance oblique est mesurée après réflexion du rayon infra-rouge sur les prismes.

Pour chaque station à terre, cel appareil est étalonné sur des points dont les coordonnées sont connues. En sonde, l'opérateur vise en permanence la couronne de prismes du bateau. La position ainsi mesurée est transmise toutes les 1,5 secondes au calculateur embarqué grâce à un émetteur radio UHF.

Cette méthode de localisation nécessite que la station à terre "voit" l'embarcation. Aussi, à cause de la forme complexe de la retenue, il a fallu implanter quinze stations de localisation sur les berges, pour pouvoir
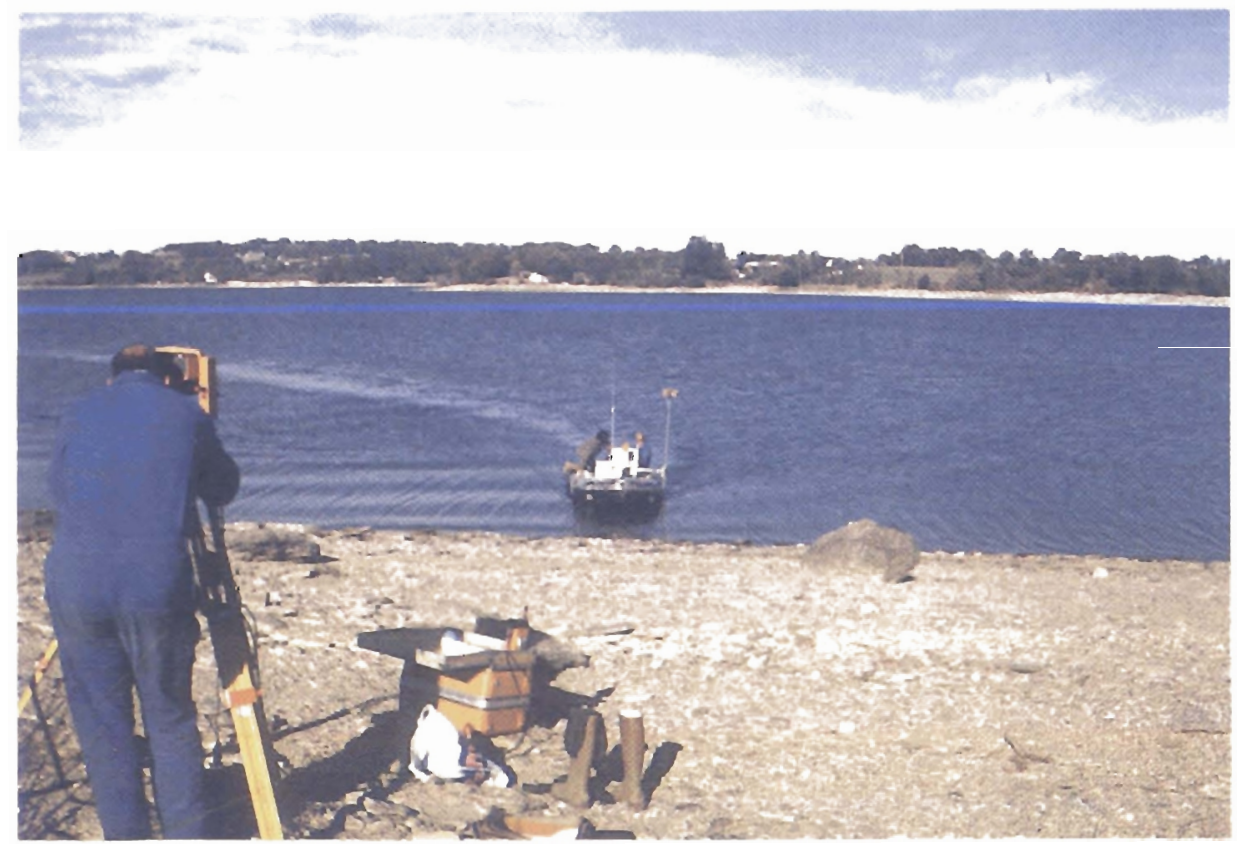

Photo 4. - Le suivi de profil.

Photo 4. - Profile reading. 
positionner le bateau dans toutes les zones. Certaines de ces stations ont pu être positionnées par visée directe à partir de la borne IGN $n^{\circ} 48$ située au lieu-dit Puech Montgrand. A partir de ces stations principales, des stations secondaires ont été positionnées pour permettre la localisation du bateau au fond des anses.

\section{III.3 Altitude du plan d'eau}

La bathymétrie s'étendant sur dix jours, la cote du plan d'eau a été mesurée régulièrement. Un repère a été installé devant la base EDF de Charouzech et coté par rapport à l'échelle limnimétrique du barrage. La hauteur du plan d'eau a été vérifiée par une visée sur le repère $n^{\circ} 10$ scellé dans le parapet du Pont des Vernhes, à l'opposé du barrage.

Pendant toute la durée du levé bathymétrique, le niveau du plan d'eau est resté pratiquement stable à la cote 801,20 mètres : au cours d'une journée, la fluctuation de niveau la plus grande a atteint $6 \mathrm{~cm}$.

\section{III.4 Bathymétrie}

Pour réaliser la bathymétrie, le lac a été divisé en 4 zones, pour lesquelles le tracé des routes (ou profils) théo-

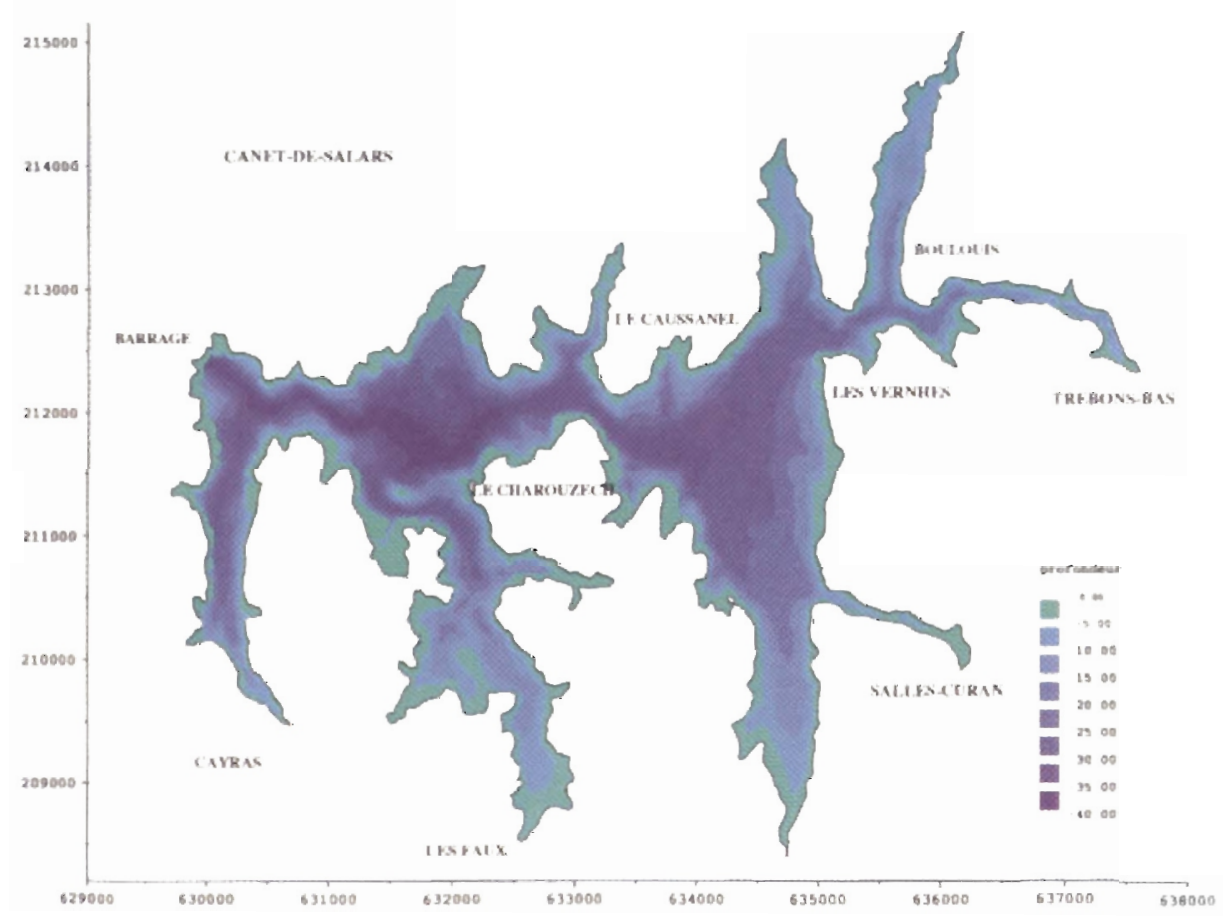

Fig. 2. - Courbes isobathes de la retenue de Pareloup.

Fig. 2. - Isobath curves for Pareloup reservoir. 
riques a été mémorisé sur le calculateur du bateau. Le suivi de ces routes sur le terrain se décompose en deux phases, l'approche et le suivi du profil.

\section{III.4.1 L'approche}

Le calculateur embarqué reçoit en permanence les paramètres de l'AGA et calcule en temps réel la position de l'embarcation. Sur l'écran du calculateur sont visualisés la route suivie par le bateau et le tracé du profil théorique recherché. Le barreur peut ainsi se positionner sur le point correspondant au début du profil théorique. Il passe alors en "suivi de profil".

\section{III.4.2 Le suivi de profil}

L'acquisition des données n'a lieu que pendant cette phase. Le barreur doit maintenir constante la vitesse du bateau et, en même temps, corriger sa route pour se rapprocher au mieux du profil théorique. Le tracé instantané, sur l'écran du calculateur, de la position du bateau permet à un barreur expérimenté de ne pas s'éloigner de plus de $2,5 \mathrm{~m}$ de part et d'autre du profil théorique.

\section{III.5 Les résultats}

Pour les besoins de l'étude, la géométrie des fonds doit être connue jusqu'à la cote maximale $805 \mathrm{~m}$. Comme la cote du plan d'eau lors du sondage était aux environs de $801,20 \mathrm{~m}$, il aurait fallu, en toute rigueur, prolonger chaque extrémité de profil par une topographie jusqu'à la cote $805 \mathrm{~m}$. Une

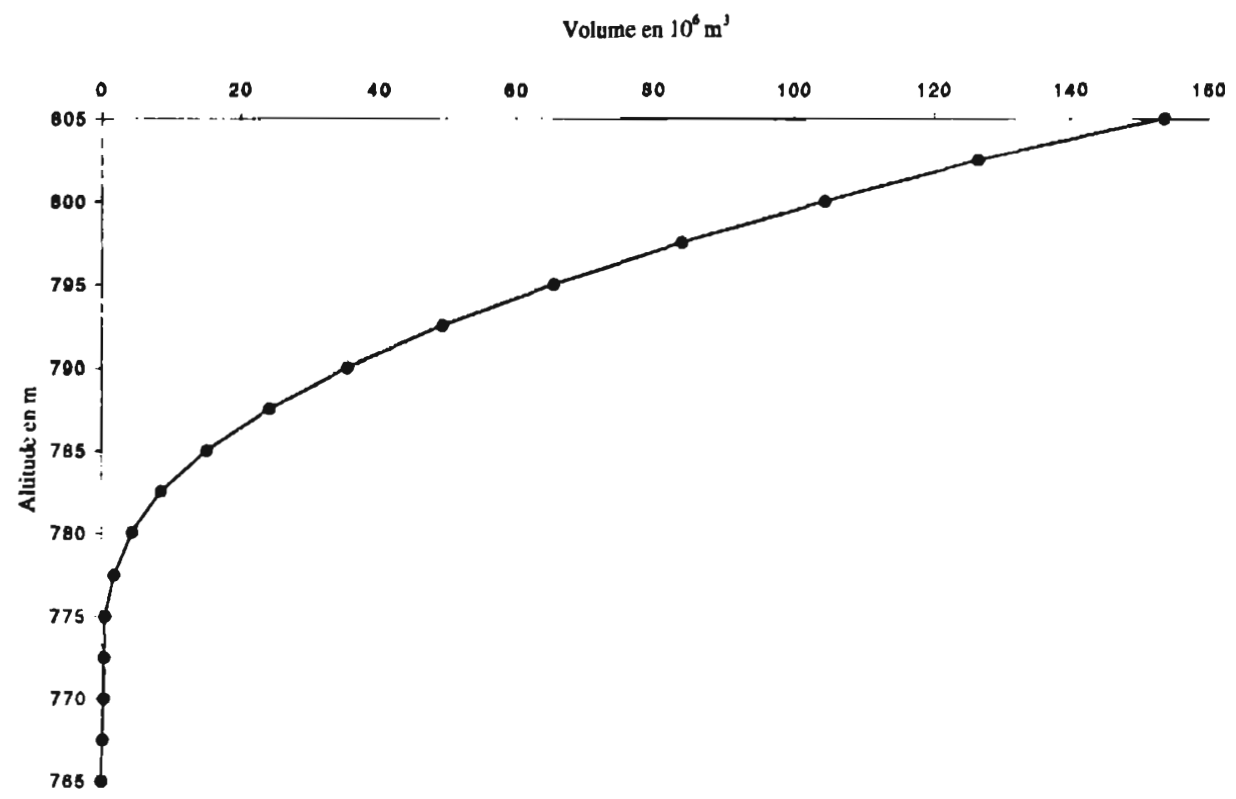

Fig. 3. - Courbe de capacité (volume-profondeur) de la retenue de Pareloup.

Fig. 3. - Capacity curve (volume-depth) for Pareloup reservoir. 
telle opération sur 180 profils n'était pas envisageable. Aussi, à partir du tracé relevé par l'IGN à la cote $805 \mathrm{~m}$, avons-nous procédé par interpolation linéaire. Nous reviendrons sur ce point ultérieurement.

Les résultats des minutes de sonde ont été traités pour obtenir les informations souhaitées :

- le tracé des isobathes sur un agrandissement IGN (1/25000) (fig. 2) ;

- un plan des abords du barrage à l'échelle $1 / 5000$;

- le calcul de la courbe de capacité (volume-profondeur) (fig. 3).

\section{REMARQUES ET CONCLUSION}

Cette bathymétrie a représenté un travail important qui appelle quelques remarques.

Le choix d'une embarcation pneumatique de type Zodiac limite l'espace de travail à bord. Cependant, ce bateau est très manœuvrable dans les petits fonds et permet des relevés jusqu'à $40 \mathrm{~cm}$ sous la surface. L'inconvénient de ce type de bateau sans quille est la dérive par grand vent.

La localisation en temps réel effectuée par poursuite optique avec le géodimètre est très précise mais contraignante, car elle nécessite plu- sieurs stations à terre et un opérateur à temps complet.

Enfin, certaines contraintes techniques ne nous ont pas permis de choisir la meilleure période pour un levé bathymétrique. En effet, la période la plus propice pour obtenir le maximum d'information correspond à celle où la cote est maximale, c'est-à-dire généralement en été. Or l'été comportait trois inconvénients majeurs pour la mise en œuvre de cette bathymétrie :

- la végétation est abondante sur les berges, ce qui gêne les visées et entraîne des stations relais supplémentaires ;

- l'été est la période où la stratification thermique est maximale dans un lac, ce qui altère la propagation ultra-sonore ;

- à cela s'ajoute la présence de nombreux touristes pratiquant des activités nautiques intenses en été.

Un compromis entre ces contraintes nous a conduits à opérer au début de l'automne, lorsque le lac est mélangé et la saison touristique terminée mais avant que ne commencent les grands creux hivernaux. Malheureusement, le turbinage d'Alrance reprend dès septembre, et c'est la raison pour laquelle la tranche de surface restera mal estimée dans cette bathymétrie. 